\title{
Posttraumatic Stress Disorder, Depression and Fear Of COVID-19 Among Pregnant Women During the Rapid Rise Period of the Coronavirus Pandemic in a Middle Eastern Country: A Cross-Sectional Study
}

\author{
Zainab Barakat \\ Lebanese University: Universite Libanaise https://orcid.org/0000-0002-6562-2914 \\ Youssef Fares \\ Lebanese University: Universite Libanaise \\ Linda Abou-Abbas ( $\sim$ l.abouabbas@ul.edu.lb) \\ Lebanese University https://orcid.org/0000-0001-9185-3831
}

\section{Research}

Keywords: COVID-19, pregnant women, PTSD, depression, fear, Lebanon.

Posted Date: June 23rd, 2021

DOl: https://doi.org/10.21203/rs.3.rs-634028/v1

License: (c) (1) This work is licensed under a Creative Commons Attribution 4.0 International License.

Read Full License 


\section{Abstract \\ Background}

Coronavirus disease 2019 (COVID-19) is an emerging public health issue. Pregnant women are susceptible to its negative psychological impact. Particularly, pre-natal psychological health is critical as it can affect the well-being of the mothers and their fetuses. The present study aimed to assess the Covid19 psychological impact on pregnant women, including posttraumatic stress disorder (PTSD), depression, and fear related to COVID-19 as well as to investigate their associated factors.

\section{Methods}

A cross-sectional study was conducted among pregnant women in Lebanon between 17th November 2020 and 27th February 2021. A standardized questionnaire was used to collect data about participants' baseline characteristics and psychological status, including PTSD, depression as well as fear related to COVID-19.

\section{Results}

Out of the 466 respondents, 28.5\% $(n=133)$ had PTSD related to Covid-19, and $22.1 \%(n=133)$ had depression. The mean score for the fear of Covid-19 was $18.51 \pm 5.55$. Pregnant women's depression and fear of Covid-19 were significantly associated with PTSD (adjusted OR $=5.85$ with $95 \% \mathrm{Cl}$ of 3.37 to 10.16 ; adjusted $\mathrm{OR}=1.19$ with $95 \% \mathrm{Cl}$ of 1.13 to 1.26 , respectively). Furthermore, smoking during pregnancy and encountering difficulties in accessing healthcare during the pandemic were found to be associated with depression, while practicing home quarantine was found to be associated with the fear of Covid-19. Pregnant women who perceived high risks towards Covid-19 and those who experienced PTSD had high levels of both depression and the fear of Covid-19 compared to their counterparts.

\section{Conclusion}

High levels of PTSD, depression, and fear of Covid-19 were detected among Lebanese pregnant women. This recommends further psychological interventions to mitigate these levels.

\section{Background}

The coronavirus disease 2019 (COVID-19) is an emerging serious public health issue that has drastically affected the lives of most people around the globe. This novel infectious disease first emerged in Wuhan, Hubei Province, China, in December 2019. Then, spread expeditiously all over the world impelling the World Health Organization to declare the COVID-19 outbreak as a worldwide pandemic [1]. 
Disease outbreaks have been suggested to have a potentially negative emotional impact on pregnant women [2]. Compared to the pre-Covid-19 period, pregnant women assessed during the Covid-19 pandemic displayed high levels of anxiety and depressive symptoms in addition to symptoms of posttraumatic stress disorder [3]. Furthermore, in a survey aiming to investigate the fear of Covid-19 among pregnant and infertile patients in Japan, Asai et al found that Japanese pregnant women had a higher fear of Covid-19 scores than Japanese infertile patients [4].

Pre-natal psychological health can affect the neurobehavioral development of the fetus and, therefore, the outcomes for the child [5]. Premature brain development was assumed in children of mothers who experienced higher perinatal depressive symptoms [6], and slower growth of the right and left hippocampus was detected during the first six months of life in children of mothers who reported increased anxiety during pregnancy [7]. Consequently, these long-lasting behavioral and structural changes highlight the importance of mental health assessment in pregnant women during this stressful outbreak.

Pregnant women consider pregnancy to be a risky time in their lives during which they are susceptible to a wide range of physical and psychological threats. Due to the negative emotional impact of the COVID19 pandemic on pregnant women, significant efforts are ongoing to better investigate their mental health status during this global outbreak.

In research carried out in China, low levels of PTSD, anxiety, depression, insomnia, and physical discomfort were detected among pregnant women during the Covid-19 outbreak [8]. However, in Italy,

Saccone et al reported that the Covid-19 epidemic had a moderate to severe psychological impact (PTSD symptom) on pregnant women [9].

In Lebanon; a Middle Eastern country, a high rate of infected pregnant women was reported by the Ministry of Public health of which several cases of death have occurred. So far, no studies have been conducted to assess the psychological status of this vulnerable group. Thus, the present study intends to assess the Covid-19 psychological impact on pregnant women during the rapid rise phase of the Covid19 pandemic, including PTSD related to COVID-19, depression, and fear of COVID-19 as well as to investigate their associated factors. The results of this study may provide health policymakers with relevant information on the psychological status of this group during the current pandemic.

\section{Methods}

\section{Study design and population:}

The present study is a cross-sectional study conducted in Lebanon from 17th November 2020 to 27th February 2021. Because of the strict measures imposed by the Covid-19 pandemic, the questionnaire was conducted online using a google form and the call for participation was made via social media platforms using the snowball technique. The inclusion criteria included Lebanese pregnant women with the ability to read and write. Any woman who was diagnosed with mental illness was excluded. 


\section{Ethical approval:}

Ethical approval for the study was obtained from the Institutional Review Board (IRB) committee of the Lebanese University Faculty of Medical Sciences (Reference number 62/2 /2021). All the collected data were kept confidential.

\section{Sample size calculation:}

Considering an anticipated response of $50 \%$, a confidence level of $95 \%$, a margin of error of $5 \%$, and an estimated population of 125,000 [10], the required sample size of this survey would be at least 383 ; it was computed using the online RAOSOFT sample size calculator.

\section{Instrumentation:}

To collect the data, a questionnaire was originally prepared in the English language, then translated to the native language in Lebanon (Arabic). The study questionnaire was developed following a review of literature on COVID-19 [11-14]. The survey questionnaire covered the following areas:

1. Baseline characteristics including age, residence, education, work status, income level, gestational age, gravida, miscarriage loss, smoking status, and complications during pregnancy. Furthermore, all participants were asked whether they were infected with COVID-19 and if they were quarantined during the pandemic. Also, they were questioned about whether their monthly family income had been affected by COVID-19 and whether they were following the news of the global outbreak.

2. To evaluate the risk perception of pregnant women during the COVID-19 outbreak, a risk perception questionnaire was used. Each item was scored on a 5 point-Likert scale with total scores ranging from 0 to 40 and the higher the scores, the higher the risk perception level. This questionnaire has shown good reliability [15]. The internal consistency of the items was evaluated in our sample, and Cronbach's alpha was 0.674 .

3. Assessment of PTSD: posttraumatic stress disorder related to the COVID-19 questionnaire (COVID19-PTSD) with its 19 items was used to evaluate PTSD risk related to the current COVID-19 emergency. All items were answered by referring to the previous seven days during the COVID-19 outbreak. It is scored on a $0-4$ Likert scale with a total score ranging from 0 to 80 ; a cutoff score of 26 indicates the presence of PTSD. The COVID-19-PTSD questionnaire has revealed excellent convergent validity, as well as reliability [16]. In this study, the internal consistency coefficient was excellent with a Cronbach's alpha of 0.933 .

4. Participants' depression severity level was assessed by using a self-administered questionnaire "the Patient Health Questionnaire (PHQ9)" with 9 items scored as 0 (not at all) to 3 (nearly every day) and a total score ranging from 0 (absence of depressive symptoms) to 27 (most severe depressive symptoms) (i.e., normal $=0-4$; mild $=5-9$; moderate $=10-14$; moderate to severe $=15-19$; and severe $=20-27$ ) [17]. PHQ9 is a valid and reliable tool among the Lebanese population; $\geq 10$ was adopted as the cut-off score to investigate the presence of depression [18]. The internal consistency of the items was evaluated in our sample, and Cronbach's alpha was 0.894 . 
5. Pregnant women's fear towards COVID-19 was investigated by using the seven questions of the fear of COVID-19 scale (FCV-19S). The scale requires a response on a 5-point Likert scale from 1 (strongly disagree) to 5 (strongly agree). The overall fear score is between 7 and 35 with higher scores indicating greater levels of fear towards COVID-19. The validity and consistency of the Arabic version of FCV-19S have been evaluated among the Saudi population [19]. In this study, the internal consistency coefficient of the FCV-19S was investigated, and Cronbach's alpha was 0.872 .

\section{Pilot study:}

The study was pretested in a sample of 6 pregnant women to check the clarity and readability of the questionnaire. Pregnant women did not report any problem concerning the clarity and the comprehensibility of the items. All the questions were completed within approximately 10 minutes.

\section{Data collection:}

Data collection was performed using an online survey. The first page of the online questionnaire consisted of a brief background about the study and its aims. The right of the participants to withdraw at any time in addition to their confidentiality and anonymity were respected. All individuals who were willing to participate in this study were asked to provide their agreement through informed consent.

\section{Statistical analysis:}

The collected data were entered into the statistical software SPSS (Statistical Package for Social Sciences), version 25 for analysis. Means and standard deviations (SD) for continuous variables, while frequency $(n)$ and percentages (\%) for categorical variables were calculated for descriptive purposes. Multivariate logistic regression analysis was carried out to identify factors related to PTSD and depression, whereas multiple linear regression analysis was performed to investigate factors associated with fear of Covid-19. Adjusted odds ratio and unstandardized regression coefficients $(\beta)$ with their $95 \%$ confidence intervals were computed to present the results of multivariate analysis. A p-value of less than 0.05 was considered statistically significant.

\section{Results}

\section{Characteristics of study population:}

The baseline characteristics of the participants are shown in Table 1. A total of 466 pregnant women participated in this survey. The mean age of the participants was $26.06 \pm 4.43$ years, ranged from 17 to 42 years. Of the total sample, 151 (32.4) had a monthly family income ranged from 1.000 .000 to 2.000.000 L.L. The majority of respondents $(80 \%)$ had a higher educational level (university students or postgraduate), $68.2 \%$ were unemployed and $7.9 \%$ were health care workers. More than one-third of participants (41.8\%) were in their second trimester, whereas $31.1 \%$ and $26.4 \%$ were in their first and third trimester, respectively. Additionally, $20 \%$ of pregnant women experienced complications during pregnancy. 
Concerning COVID-19, $7.1 \%$ of the participants were infected with coronavirus and $14.2 \%$ had difficulty accessing healthcare during the pandemic. Moreover, $56.9 \%$ of the pregnant women reported that the outbreak had affected their monthly family income and $86.9 \%$ indicated that they were following the news of this global epidemic.

Table 1. Baseline characteristics of the study respondents $(\mathrm{N}=466)$. 


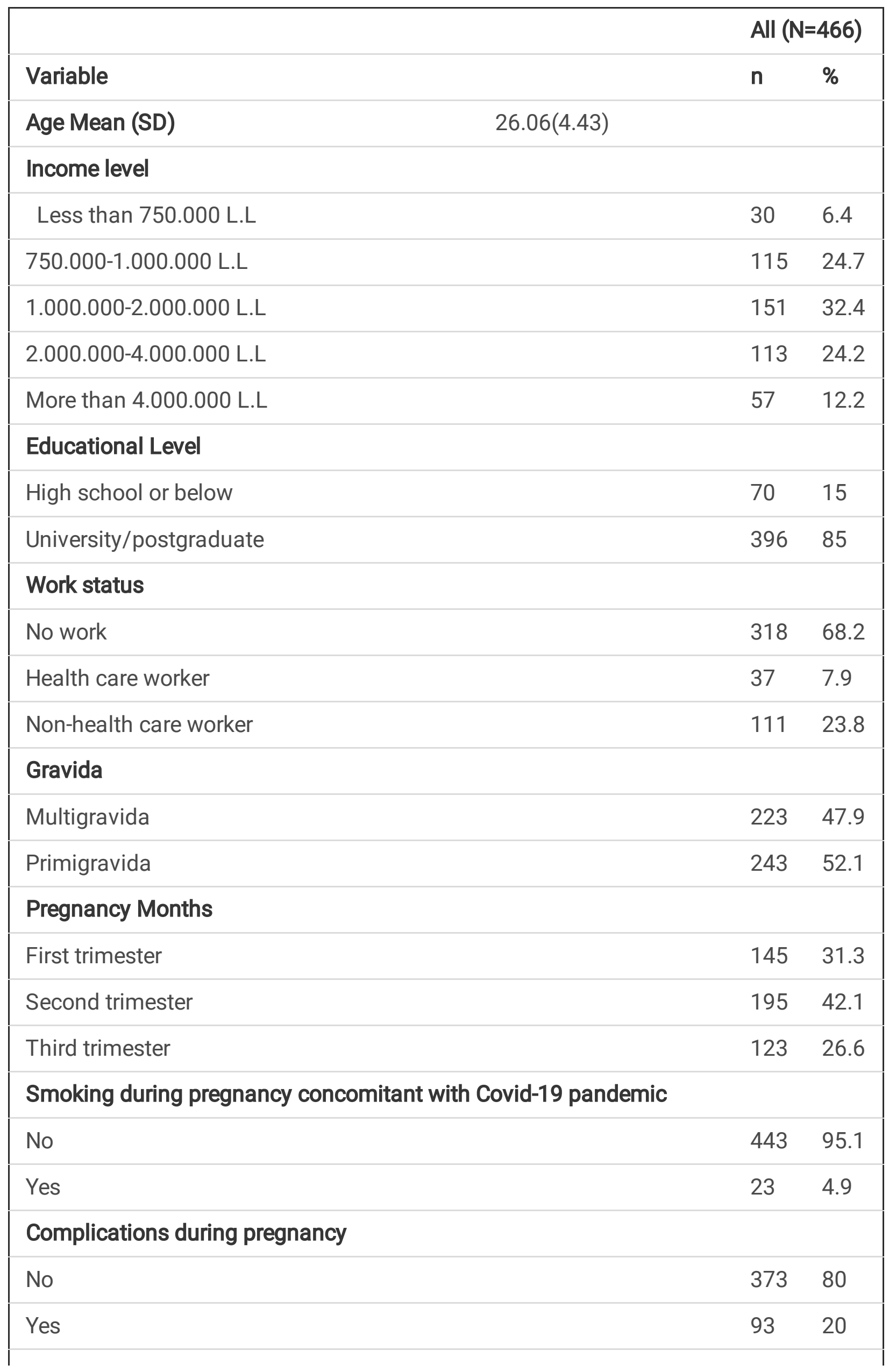




\begin{tabular}{|l|cc|}
\hline Infection with Covid-19 & 433 & 92.9 \\
\hline Yes & 33 & 7.1 \\
\hline Practicing home quarantine during Covid-19 pandemic & & \\
\hline No & 169 & 36.3 \\
\hline Yes & 297 & 63.7 \\
\hline Having a family monthly income affected by Covid-19 & & \\
\hline No & 201 & 43.1 \\
\hline Yes & 265 & 56.9 \\
\hline Encountering difficulties in accessing health care during the pandemic & & \\
\hline No & 400 & 85.8 \\
\hline Yes & 66 & 14.2 \\
\hline Following the news of the global outbreak & & \\
\hline No & 61 & 13.1 \\
\hline Yes & 405 & 86.9 \\
\hline Risk perception Mean (SD) & & \\
\hline
\end{tabular}

$\mathrm{n}$ : frequency, \%: percentage, SD: standard deviation

The levels of PTSD relative to Covid-19, depression, and the fear of Covid-19 among pregnant women

The prevalence of PTSD and depression among Lebanese pregnant women elevated during the rapid rise of Covid-19. Out of the total sample, 133 (28.5\%) had PTSD, and $103(22.1 \%)$ had depression. Stratified by severity, 14 (3\%) women were severely depressed, 26 (5.6\%) women were moderately to severely depressed, 63 women (13.5\%) were moderately depressed, and $163(35 \%)$ women were mildly depressed. Further, the mean score for the fear of Covid-19 was $18.51 \pm 5.55$. (Figure1, 2).

\section{Factors associated with PTSD symptomatology among pregnant women}

Table 2 indicates the results of multiple logistic regression analysis. Pregnant women who experienced depression were 5.85 times more likely to have PTSD compared to their counterparts (adjusted OR=5.85 with $95 \% \mathrm{Cl}$ of 3.37 to 10.16 ). The results also revealed that the fear of pregnant women towards Covid-19 was significantly associated with PTSD (adjusted OR=1.19 with $95 \% \mathrm{Cl}$ of 1.13 to 1.26 ).

Table 2. Factors associated with PTSD related to Covid-19 among pregnant women. 


\begin{tabular}{|lllll|}
\hline PTSD & No & Yes & Adjusted OR (95\% Cl) & P-value \\
\hline Depression n (\%) & & & & $<0.000$ * $^{*}$ \\
\hline No & $296(81.5)$ & $67(18.5)$ & 1 & \\
\hline Yes & $37(35.9)$ & $66(64.1)$ & $5.85(3.37,10.16)$ & \\
\hline Fear Mean (SD) & $17(4.62)$ & $22.29(5.88)$ & $1.19(1.13,1.26)$ & $<0.0001^{*}$ \\
\hline
\end{tabular}

Variables entered into the model: educational level, gravida, complications during pregnancy, practicing home quarantine, having a family monthly income affected by Covid-19, encountering difficulties in accessing healthcare during the pandemic, risk perception score, depression, and fear score. $\mathrm{n}$ frequency, $\%$ Percentage, SD standard deviation, $\mathrm{Cl}$ confidence interval, OR odds Ratio, *P-value $₫ 0.05$ is considered significant.

\section{Factors associated with depression among pregnant women}

Multivariate logistic regression analysis showed that women who smoked during pregnancy were 4.06 times more likely to suffer from depression than those who did not smoke (OR=4.06 with $95 \% \mathrm{Cl}$ of 1.51 to 10.94). As well, pregnant women who had difficulty accessing healthcare during the pandemic showed a 2.81 -fold higher probability of being depressed (OR $=2.81$ with $95 \% \mathrm{Cl}$ of 1.50 to 5.26 ). The odds of risk perception were also found to be significantly associated with depression (OR=1.11 with $95 \% \mathrm{Cl}$ of 1.03 to 1.20 respectively). Moreover, women who displayed PTSD related to Covid-19 were 5.70 times more likely to experience depression compared to their counterparts (OR=5.70 with $95 \% \mathrm{Cl}$ of 3.26 to 9.96 ). (Table 3)

Table 3. Factors associated with depression in pregnant women during the Covid-19 pandemic. 


\begin{tabular}{|c|c|c|c|c|}
\hline \multicolumn{5}{|l|}{ Depression } \\
\hline & No & Yes & $\begin{array}{l}\text { Adjusted OR } \\
(95 \% \mathrm{Cl})\end{array}$ & P-value \\
\hline Smoking during pregnancy $\mathrm{n}(\%)$ & & & & $0.006 *$ \\
\hline No & $\begin{array}{l}352 \\
(79.5)\end{array}$ & $\begin{array}{l}91 \\
(20.5)\end{array}$ & 1.00 & \\
\hline Yes & $\begin{array}{l}11 \\
(47.8)\end{array}$ & $\begin{array}{l}12 \\
(52.2)\end{array}$ & $\begin{array}{l}4.06 \\
(1.51,10.94)\end{array}$ & \\
\hline $\begin{array}{l}\text { Encountering difficulties in accessing healthcare } \\
\text { during the pandemic } n(\%)\end{array}$ & & & & $0.001 *$ \\
\hline No & $\begin{array}{l}328 \\
(82)\end{array}$ & $72(18)$ & 1.00 & \\
\hline Yes & $35(53)$ & $31(47)$ & $\begin{array}{l}2.81 \\
(1.50,5.26)\end{array}$ & \\
\hline COVID-19 Risk perception (Mean) SD & $\begin{array}{l}22.39 \\
(4.19)\end{array}$ & $\begin{array}{l}25.09 \\
(3.93)\end{array}$ & $\begin{array}{l}1.11 \\
(1.03,1.20)\end{array}$ & $0.005^{*}$ \\
\hline PTSD n (\%) & & & & $<0.0001 *$ \\
\hline No & $\begin{array}{l}296 \\
(88.9)\end{array}$ & $\begin{array}{l}37 \\
(11.1)\end{array}$ & 1.00 & \\
\hline Yes & $\begin{array}{l}67 \\
(50.4)\end{array}$ & $\begin{array}{l}66 \\
(49.6)\end{array}$ & $\begin{array}{l}5.70 \\
(3.26,9.96)\end{array}$ & \\
\hline
\end{tabular}

Variables entered into the model: gravida, smoking during pregnancy, complications during pregnancy, practicing home quarantine, having a family monthly income affected by Covid-19, encountering difficulties in accessing healthcare during the pandemic, risk perception score, fear score, and PTSD. $n$

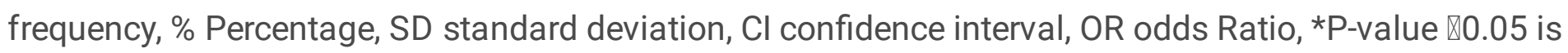
considered significant.

\section{Factors associated with fear of Covid-19 among pregnant women}

Results from multiple linear regression revealed that pregnant women who quarantined themselves during the pandemic (beta $=1.12,95 \% \mathrm{Cl}(0.28,1.95)$; P-value=0.009) experienced a high level of fear concerning Covid-19 compared to their counterparts. Moreover, the findings showed that pregnant women who perceived high risks of the disease (beta $=0.58,95 \% \mathrm{Cl}(0.48,0.68)$; P-value $<0.0001$, and those who suffered from PTSD related to Covid-19 (beta $=3.54,95 \% \mathrm{Cl}(2.61,4.46)$; P-value $<0.0001$, had high levels of fear towards Covid-19 compared to their counterparts (Table 4).

Table 4. Results of multiple linear regression on factors associated with pregnant women's fear score toward Covid-19. 


\begin{tabular}{|lllll|}
\hline Predictor & $\mathbf{b}(95 \% \mathrm{Cl})$ & SE B & Standardized $\mathrm{P}$ & P-value \\
\hline Practicing home quarantine & $1.12(0.28,1.95)$ & 0.43 & 0.10 & $\mathbf{0 . 0 0 9 *}$ \\
\hline Risk perception & $0.58(0.48,0.68)$ & 0.05 & 0.45 & $<0.0001^{*}$ \\
\hline PTSD & $3.54(2.61,4.46)$ & 0.47 & 0.29 & $<0.0001^{*}$ \\
\hline
\end{tabular}

Factors entered into the model: gravida, infection with Covid-19, complications during pregnancy, encountering difficulties in accessing healthcare during the pandemic, following the news of the global outbreak, and depression. Cl: confidence interval, SE B: standard error beta, 『: beta, * P-value less than 0.05 is considered significant.

\section{Discussion}

This cross-sectional study aimed to investigate the psychological status of pregnant women, including PTSD related to Covid-19, depression, and fear of Covid-19 during the rapid rise period of the coronavirus spread in Lebanon. In addition, it sought to identify the prevalence of PTSD and depression in this vulnerable population. The results of this survey indicated a high prevalence of PTSD and depression among Lebanese pregnant women. It also revealed that women who experienced depression and displayed a high score of the fear of Covid-19 had a higher level of PTSD related to Covid-19 compared to their counterparts. In addition, smoking during pregnancy and encountering difficulties in accessing healthcare during the pandemic were found to be associated with depression, while practicing home quarantine was detected to be related to fear of Covid-19. Interestingly, pregnant women who perceived high risks towards Covid-19 and those who experienced PTSD had high levels of both depression and the fear of Covid-19 compared to their counterparts.

In the present study, $28.5 \%$ of pregnant women were suffering from PTSD related to Covid-19. This result is relevant to the findings obtained by a study conducted in Italy, where $29.5 \%$ of the Italian population suffered from PTSD; it's of utmost importance to mention that this study is the one that validated the PTSD-COVID-19 questionnaire and indicated a cutoff score of 26 that was used in our survey [11]. The prevalence of PTSD in a study carried out among pregnant women from all regions in China during the early phase of the Covid-19 pandemic (from 13 to 16 February 2020), was $40 \%$, which is higher than the one obtained in our survey [20]. However, another study conducted among the Chinese pregnant women during the reducing stage after the Covid-19 epidemic's peak in China (from February 28 to March 12), revealed a percentage of PTSD of $0.9 \%$, which is much lower than that found in our study [8]. This discrepancy in the prevalence estimates might be attributed to cultural differences and pandemic phases.

Multiple logistic regression analysis showed that pregnant women who experienced depression and had a high score of the fear of Covid-19 displayed a higher level of PTSD than their counterparts. This indicates that participants with high levels of depression and fear related to Covid-19 are at an increased risk of developing PTSD. A concurrent diagnosis of PTSD and depression was demonstrated in a study carried out by Gros et al [21]. Interestingly, our survey demonstrated fear as a predictor of PTSD, but not 
of depression; this is consistent with the result of research that showed impaired fear inhibition as a biomarker of PTSD but not depression [22]. Therefore, these data demonstrated a negative emotional impact of Covid-19 on pregnant women that calls for the need to evaluate the psychiatric characteristics of such a group during the Covid-19 period. Also, it entails the urgent need for psychological interventions to enhance the mental health status of pregnant women and avoid any complications that may affect the well-being of themselves and their fetuses.

Concerning depression, approximately $22 \%$ of the participants were suffering from depression during the Covid-19 pandemic; this prevalence is in line with the one found among healthcare workers at a tertiary care hospital in Lebanon [23]. Even though it seems to be lower than the results obtained in a study conducted among pregnant women in Sari and Amol, Iran, it is higher than the one found among Sri Lanka pregnant women $[24,25]$. One of the possible explanations of the high depression level found in our results is the fact that Covid-19 prevention entails social distancing, home quarantine, and curfews which could result in social isolation, worries, fear, exasperation, and sorrow. Logistic regression analysis showed that women who smoked while pregnant are at an increased risk of experiencing depression; in agreement with our findings, a study conducted among pregnant Syrian refugees and pregnant citizens of the Republic of Turkey has found a statistically significant correlation between smoking status and risk for depression [26]. This suggests the need for awareness interventions about smoking during pregnancy. Additionally, pregnant individuals who had difficulty accessing healthcare during the pandemic were more likely to suffer from depression compared to their counterparts; this indicates that restricted movements or obstacles imposed by the Covid-19 pandemic have contributed in part to the increase of pregnant women's depression level. Perceiving high risks toward coronavirus was also significantly associated with depression; this result is supported by the findings obtained in a large-scale cross-country study of the psychological impact of COVID-19 [27]. Finally, pregnant women who were suffering from PTSD related to Covid-19 displayed higher levels of depression, in contrast to those without PTSD; this finding is consistent with a study conducted among pregnant women in China, which showed that pregnant women with suspected PTSD were more likely to develop prenatal depression than their counterparts [20]. Also, Gros et al found a previous or current depression in $48 \%$ to $55 \%$ of individuals who have been diagnosed with PTSD [21]. Thus, this necessitates paying attention to the mental health status of pregnant women during the current pandemic.

Moreover, our study found that the mean score of the fear of Covid-19 among pregnant women was $18.51 \pm 5.55$. Although this finding is higher than the one obtained in Eastern India [28], it is lower than that computed in Turkey [29]. Multiple linear regression analysis revealed that practicing home quarantine, perceiving high risks toward Covid-19, and suffering from PTSD related to Covid-19 are the predictors of the fear of Covid-19, with risk perception being considered as the best predictor ( $\mathrm{P}$ value $<0.0001$, Beta=0.45). The rapid spread of the Covid-19 pandemic in Lebanon, accompanied by the increasing rate of Covid-19 death, especially among pregnant women could explain the increased level of fear observed among these pregnant individuals. 
The current study has some limitations. Due to its cross-sectional nature, we are not able to make a cause-effect relationship. Also, the individuals were not randomly selected for participation making our findings not generalizable. Another limitation is that not all used scales have been validated in Arabic. Furthermore, this study was carried out at a particular point in time, which demonstrates the need for longitudinal studies to follow up on the psychological status of pregnant women during this pandemic.

\section{Conclusion}

The present study revealed important insights into pregnant women's psychological status, including PTSD related to Covid-19, depression, and the fear of Covid-19. Lebanese pregnant women showed high levels of PTSD, depression, and fear during the rapid rise period of the coronavirus pandemic in Lebanon. This recommends the implementation of psychological interventions to enhance their mental health status and avoid any complications that may affect the well-being of them and their fetus.

\section{Abbreviations}

COVID-19: Coronavirus disease 2019

Covid-19: Coronavirus disease 2019

PTSD: Posttraumatic stress disorder

IRB: Institutional Review Board

PHQ9: The Patient Health Questionnaire

FCV-19S: The Fear of COVID-19 Scale

OR: Odds Ratio

Cl: Confidence Interval

SPSS: Statistical Package for Social Sciences

SD: Standard Deviation

\section{Declarations}

\section{Ethics approval and consent to participate}

Ethical approval was obtained by the scientific research committee of the Neuroscience Research Center, Faculty of Medical Sciences at the Lebanese University (Reference number 62/2 /2021). Participants had to answer a yes-no question to confirm their willingness to participate voluntarily. All the necessary 
measures to safeguard participants' anonymity and confidentiality of information were respected. Written informed consent approved by the ethics committee was obtained from all the participants.

\section{Consent for publication}

Not applicable.

\section{Availability of data and materials}

Data are available from the corresponding authors upon reasonable request.

\section{Competing interests}

The author(s) declare that they have no competing interests.

\section{Funding}

No funding was received.

\section{Author's contributions}

ZB and LAA developed the project idea, performed the literature review, formulated the questionnaire, organized and analyzed the survey, drafted and critically reviewed the paper. YF reviewed the manuscript for important intellectual content. All authors read and approved the final manuscript.

\section{Acknowledgements}

The authors would like to thank all pregnant women who accepted to participate in this study.

\section{Author's information}

\section{Affiliations}

Faculty of Medical Sciences, Neuroscience Research Center, Lebanese University, Hadath, Lebanon

Zainab Barakat, Linda Abou-Abbas \& Youssef Fares

Corresponding author

Correspondence to Linda Abou-Abbas.

\section{References}

1. World Health Organization. WHO announces COVID-19 outbreak a pandemic.. 202013 March 2021]; Available from: https://www.euro.who.int/en/health-topics/health-emergencies/coronavirus-covid19/news/news/2020/3/who-announces-covid-19-outbreak-a-pandemic. 
2. Brooks SK, Weston D, Greenberg N, Psychological impact of infectious disease outbreaks on pregnant women: Rapid evidence review. medRxiv, 2020: p. 2020.04.16.20068031.

3. Berthelot $\mathrm{N}$, et al. Uptrend in distress and psychiatric symptomatology in pregnant women during the coronavirus disease 2019 pandemic. Acta Obstet Gynecol Scand. 2020;99(7):848-55.

4. Asai K, et al. Fear of novel coronavirus disease (COVID-19) among pregnant and infertile women in Japan. Journal of Affective Disorders Reports. 2021;4:100104.

5. Kinsella MT, Monk C. Impact of maternal stress, depression and anxiety on fetal neurobehavioral development. Clin Obstet Gynecol. 2009;52(3):425-40.

6. Lebel C, et al. Prepartum and Postpartum Maternal Depressive Symptoms Are Related to Children's Brain Structure in Preschool. Biol Psychiat. 2016;80(11):859-68.

7. Qiu A, et al. Maternal anxiety and infants' hippocampal development: timing matters. Transl Psychiatry. 2013;3(9):e306.

8. Zhou Y, et al. The prevalence of psychiatric symptoms of pregnant and non-pregnant women during the COVID-19 epidemic. Translational Psychiatry. 2020;10(1):319.

9. Saccone G, et al. Psychological impact of coronavirus disease 2019 in pregnant women. American journal of obstetrics gynecology. 2020;223(2):293-5.

10. KAK FE, COVID-19 AND PREGNANCY: LEBANON PREPAREDNESS WITHIN GLOBAL RESPONSE. 2020.

11. Forte G, et al. COVID-19 Pandemic in the Italian Population: Validation of a Post-Traumatic Stress Disorder Questionnaire and Prevalence of PTSD Symptomatology. Int J Environ Res Public Health. 2020;17(11):4151.

12. Ahorsu DK, et al. The Fear of COVID-19 Scale: Development and Initial Validation. International Journal of Mental Health and Addiction; 2020.

13. Centers for Disease Control and Prevention. Breastfeeding and Caring for Newborns. 202015 November 2020]; Available from: https://www.cdc.gov/coronavirus/2019-ncov/need-extraprecautions/pregnancy-breastfeeding.html.

14. World Health Organization. Coronavirus disease (COVID-19): Pregnancy and childbirth. 20209 November 2020]; Available from: https://www.who.int/news-room/q-a-detail/coronavirus-diseasecovid-19-pregnancy-and-childbirth.

15. Yue $\mathrm{C}$, et al., Association between social support and anxiety among pregnant women in the third trimester during the coronavirus disease 2019 (COVID-19) epidemic in Qingdao, China: The mediating effect of risk perception. The International journal of social psychiatry, 2020: p. $20764020941567-20764020941567$.

16. Forte G, et al., COVID-19 Pandemic in the Italian Population: Validation of a Post-Traumatic Stress Disorder Questionnaire and Prevalence of PTSD Symptomatology. Int J Environ Res Public Health, 2020. 17(11).

17. Kroenke K, et al. The Patient Health Questionnaire Somatic, Anxiety, and Depressive Symptom Scales: a systematic review. Gen Hosp Psychiatry. 2010;32(4):345-59. 
18. Sawaya $\mathrm{H}$, et al. Adaptation and initial validation of the Patient Health Questionnaire - 9 (PHQ-9) and the Generalized Anxiety Disorder - 7 Questionnaire (GAD-7) in an Arabic speaking Lebanese psychiatric outpatient sample. Psychiatry Res. 2016;239:245-52.

19. Alyami M, et al., Psychometric Evaluation of the Arabic Version of the Fear of COVID-19 Scale. Int J Ment Health Addict, 2020: p. 1-14.

20. Zhang CJP, et al. Psychobehavioral Responses, Post-Traumatic Stress and Depression in Pregnancy During the Early Phase of COVID-19 Outbreak. Psychiatric Research Clinical Practice. 2020;3(1):4654.

21. Gros DF, et al. Symptom overlap in posttraumatic stress disorder and major depression. Psychiatry Res. 2012;196(2):267-70.

22. Jovanovic T, et al. Impaired fear inhibition is a biomarker of PTSD but not depression. Depress Anxiety. 2010;27(3):244-51.

23. Khoury ME. F., et al., Factors Associated with Mental Health Outcomes: Results from a Tertiary Referral Hospital in Lebanon during the COVID-19 Pandemic. Libyan Journal of Medicine. 2021;16(1):1901438.

24. Wu F, et al. Prevalence and contributory factors of anxiety and depression among pregnant women in the post-pandemic era of COVID-19 in Shenzhen, China. J Affect Disord. 2021;291:243-51.

25. Patabendige $M$, et al. Psychological impact of the COVID-19 pandemic among pregnant women in Sri Lanka. International Journal of Gynecology Obstetrics. 2020;151(1):150-3.

26. Kurtuluş Ş, Can R, Sak ZHA. Assessment of the Relationship Between Smoking and Depression in Pregnant Women. J Immigr Minor Health. 2021;23(3):536-46.

27. Han Q, et al. Associations of risk perception of COVID-19 with emotion and mental health during the pandemic. J Affect Disord. 2021;284:247-55.

28. Sahu DP, et al. Fear and anxiety among COVID-19 Screening Clinic Beneficiaries of a tertiary care hospital of Eastern India. Asian J Psychiatr. 2021;57:102543.

29. Bakioğlu F, Korkmaz O, Ercan H. Fear of COVID-19 and Positivity: Mediating Role of Intolerance of Uncertainty, Depression, Anxiety, and Stress. International Journal of Mental Health and Addiction; 2020.

\section{Figures}




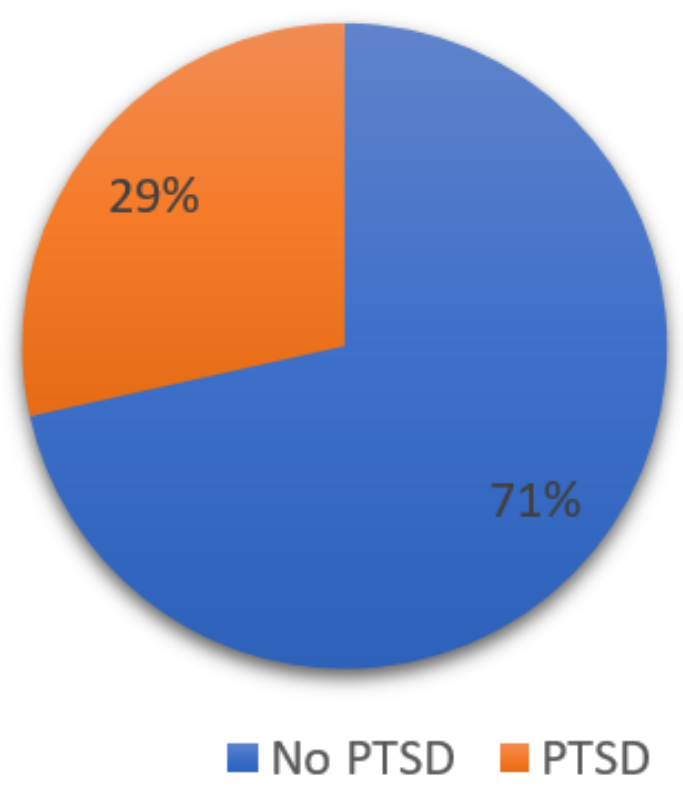

Figure 1

The prevalence of PTSD among pregnant women during the Covid-19 pandemic.

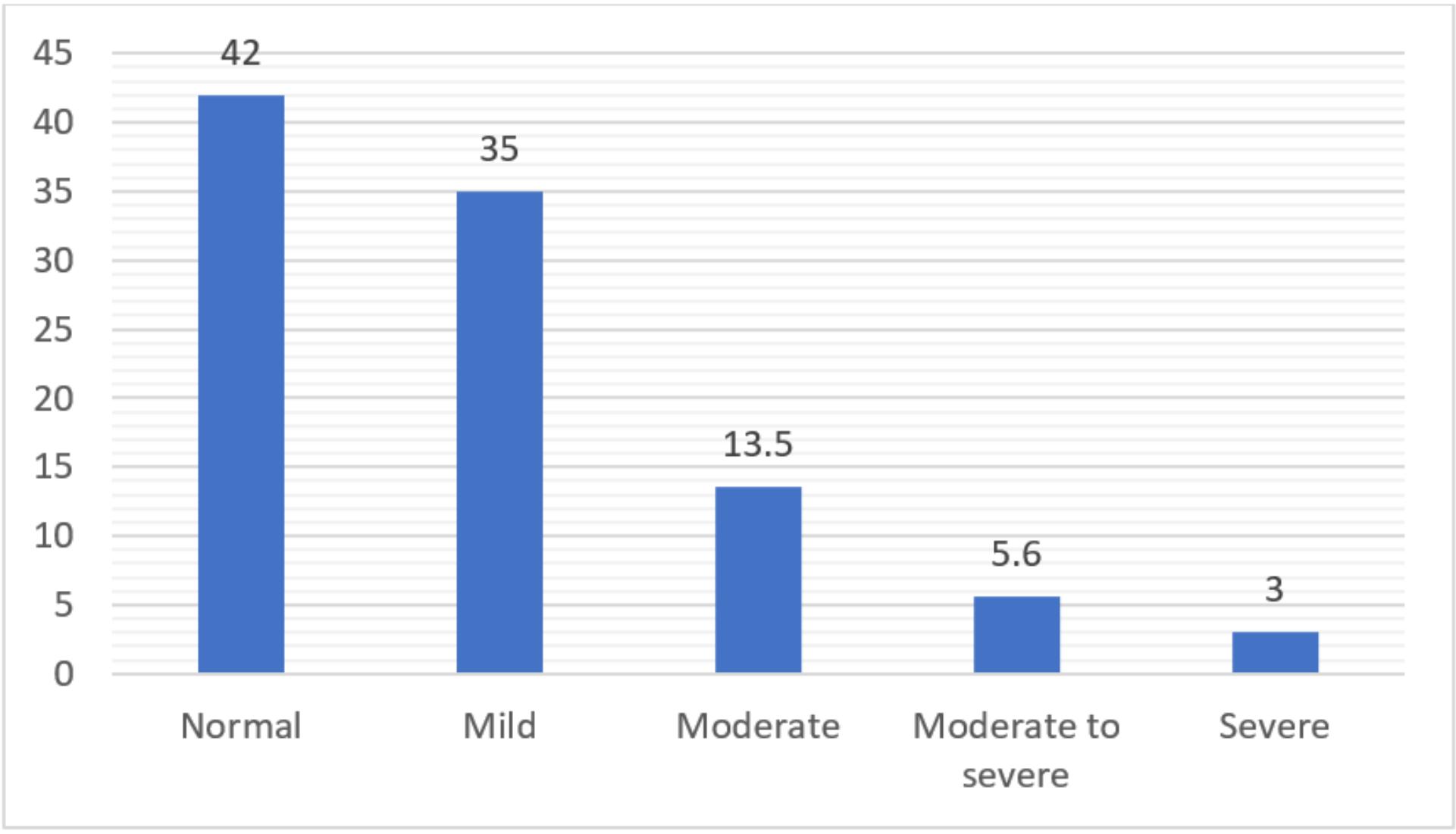

Figure 2 
The severity of depression in pregnant women during the Covid-19 pandemic. 